\title{
Descrição de adaptação da técnica de coleta de sangue por punção cardíaca em serpentes, através do estudo em cascavéis (Crotalus durissus)
}

Description of technique adaptation for blood collection through cardiac puncture in serpents through a study in rattle snakes (Crotalus durissus)

\author{
Laura Nunes ${ }^{\dagger *}$, Ana Paula Martinez de Abreu ${ }^{\ddagger}$,Alvaro Alberto Moura Sá dos Passos $\$$, Fabio Sartori", \\ Gustavo Silveira Gonçalves ${ }^{\S}$, Bernadete Maria De Sousall
}

\begin{abstract}
Resumo
Apadronização das técnicas de punção para coleta de sangue em serpentes é um corrente desafio na clínica de animais selvagens. O presente trabalho propõe uma técnica modificada de punção cardíaca em Crotalus durissus (Cascavel). Normalmente, as veias coccígeas caudais e palatinas, ou os ramos espinhais dorsais das veias espinhais dorsolaterais, são os locais mais utilizados para a coleta de sangue em serpentes. Entretanto se mostram pouco eficientes em cascavéis quando há necessidade de amostras sanguíneas acima de $1 \mathrm{~mL}$. A técnica descrita propõe a coleta na base do coração, que pode ser localizado por meio de palpação, sendo reconhecida pela forte pulsação local, e em posição lateralizada pelo lado direito, no espaço entre as junções das sexagésima oitava escama ventral com as escamas láterodorsais, inserindose a agulha cuidadosamente em uma angulação de $90^{\circ}$. O Sangue deve ser retirado lentamente para não promover o colabamento das paredes cardíacas. Esta técnica foi reproduzida em cinco indivíduos, que após serem submetidos à coleta apresentaram normalidade em seus padrões clínicos.
\end{abstract}

Como citar esse artigo. Nunes L, de Abreu APM, dos Passos AAMS, G onçalves GS, de Sousa BM. Descrição de adaptação da técnica de coleta de sangue por punção cardíaca em serpentes, através do estudo em cascavéis (Crotalus durissus). Revista de Saúde. 2017 Jan./Jun.; 08 (1): $22-24$.
Palavras-chave: Coleta de sangue; Animais Silvestres; Serpentes.

\begin{abstract}
Standardization of puncture techniques for blood collection in snakes poses a challenge in wild animal practice. This paper presents a modified technique for cardiac puncture in Crotalus durissus (rattlesnake), which is safer and yields a larger amount of blood. Usual choices for blood collection include coccygeal veins and palate, or dorsal spinal branches of the dorsolateral spinal veins; however they are inefficient when blood samples in excess of $1 \mathrm{~mL}$ are required, especially in rattlesnakes. The technique described here proposes puncture of the heart base, ventrally, which can be recognized by the presence of a strong local pulse, and laterally, on the right side, on the space between the sixty-eighth ventral scale and its junction with the dorsolateral scales, followed by careful insertion of the collection needle at a $90^{\circ}$ angle. Blood should be removed slowly to prevent collapse of cardiac walls. This technique was reproduced in five individuals, who presented normal behavior and normal clinical variables after the collection.

Keywords: Blood collection; Wild animals; Snakes.
\end{abstract}

\section{Introdução}

O constante desafio para o médico veterinário na clínica de animais selvagens é proporcional à grande variedade de espécies e suas particularidades, que normalmente trazem a necessidade de inúmeras e inusitadas adaptações de técnicas, como por exemplo, técnicas de coleta sanguínea, comumente utilizadas na medicina veterinária. Os exames hematológicos e bioquímicos, assim como testes sorológicos possuem grande relevância no diagnóstico clínico de serpentes ${ }^{1}$. De acordo com estas considerações, o presente artigo tem o intuito de descrever uma adaptação utilizada para técnica de punção cardíaca em serpentes. Normalmente a coleta de sangue em serpentes é feito por acesso à veia coccígea caudal e palatina $^{1}$, por acesso aos ramos espinhais dorsais das veias espinhais dorsolaterais ${ }^{2}$ ou, ainda, por meio de cardiocentese, diretamente no ventrículo, localizado através de estudos de folidose ${ }^{1-3}$, sendo necessário conhecer a posição do órgão cardíaco em relação as escamas ventrais em cada espécie ${ }^{1}$.

As punções palatinas, coccígea caudal e espinhais se mostraram pouco eficientes em coletas com volume acima de $1 \mathrm{~mL}$, situação que depende do tamanho de cada animal. Levando em consideração outros fatores importantes como a fisiologia cardiocirculatória e metabólica das serpentes, essas dificuldades levaram à opção pela punção cardíaca como método de coleta sangue, permitindo a coleta de maior volume de material biológico, e sendo

Afiliação dos autores: † Discente docurso de Medicina Veterinária da Universidade Severino Sombra (USS), Vassouras, RJ, Brasil

\ Docentes do curso de Medicina Veterinária da Universidade Severino Sombra (USS), Vassouras, RJ, Brasil

§ Discente do programa de Pós-Graduação da Universidade Federal de Juiz de Fora (UFJF), Juiz de Fora, MG, Brasil

|| Docente do programa de Pós-Graduação da Universidade Federal de Juiz de Fora (UFJF),Juiz de Fora, MG, Brasil

* Endereço para correspondecia: Universidade Severino Sombra (USS), Av. Expedicionário Oswaldo de Almeida Ramos, n 390, Centro - Vassouras / RJ CEP: 27700-000, (24)

24717992, lauranunes123@hotmail.com 
utilizada em aves e répteis ${ }^{4-6}$. A cardiocentese é também o método de escolha quando as serpentes possuem menos de 1 metro de comprimento ${ }^{7}$.

\section{Material e métodos}

Com o animal não sedado e devidamente contido pela região ventral, no terço proximal à cabeça $^{8}$ e por meio de palpação com as pontas dos dedos, localiza-se a base do coração, que é reconhecida pela forte pulsação local, em posição lateralizada pelo lado direito, no espaço entre as junções das sexagésima oitava escama ventral com as escamas látero dorsais. A higienização do local é realizada com álcool 70\%. Após a limpeza, deve-se cuidadosamente rebater as escamas ventrais com a agulha e inserir a mesma em uma angulação de $90^{\circ}$, e lentamente transpassar a musculatura até atingir o coração. A confirmação do posicionamento correto será constatada pela forte sensibilidade da pulsação cardíaca, retirando o sangue lentamente para não promover o colabamento das paredes cardíacas. Utiliza-se seringa com agulha hipodérmica, estéril, descartável de $1 \mathrm{~mL}$, com agulha $0,13 \mathrm{~mm}$ x $4,5 \mathrm{~mm}$, como pode ser visto na Figura 1. A contenção e a angulação da agulha para a punção podem ser vistas na Figura 2.

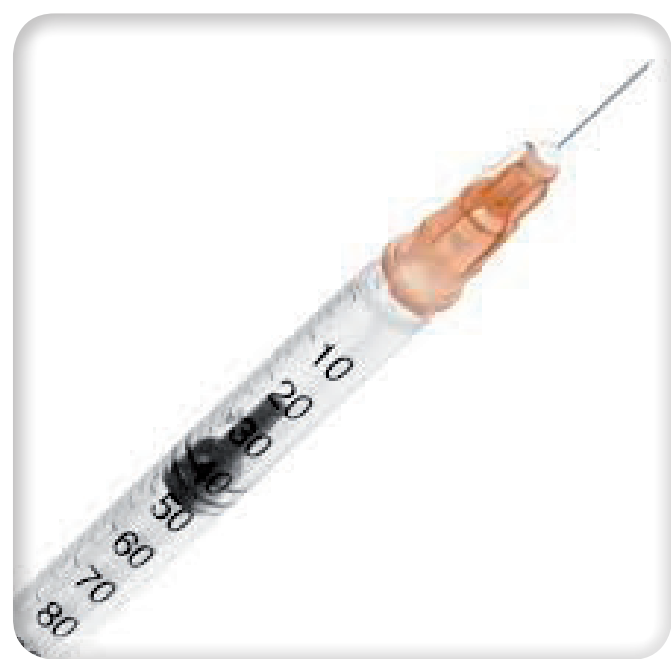

Figura 1. Seringa e agulha utilizadas na coleta. Fonte: Próprios autores.
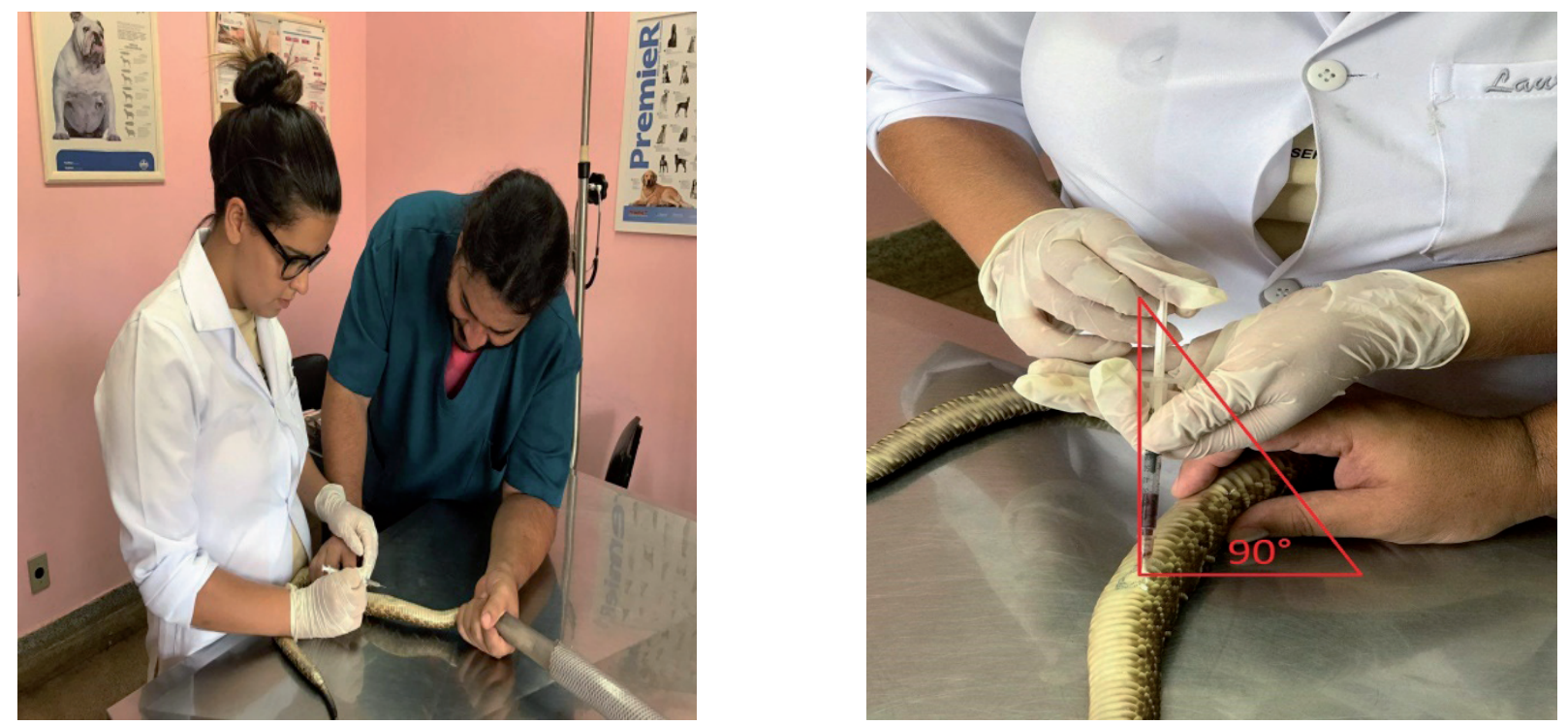

Figuras 2. Na foto da esquerda contenção da serpente na posição dorso ventral identificando no terço proximal à cabeça o frêmito cardíaco para identificação do coração. Na foto da direita após rebater escamas ventrais angulando a agulha à $90^{\circ}$ iniciando a punção cardíaca. Fonte: Próprios autores. 


\section{Resultados}

Foram coletados cinco animais, sendo todas cascavéis, capturadas pelo corpo de bombeiros e levadas para o Hospital Veterinário da Universidade Severino Sombra. Em média foram obtidos $1,3 \mathrm{~mL}$ de sangue, com mínimo de $0,7 \mathrm{~mL}$ e máximo de $2 \mathrm{~mL}$, situação onde duas seringas precisaram ser utilizadas. As punções foram bem sucedidas, sem apresentação de efeitos adversos após o procedimento, tendo sido os animais acompanhados por um período de 6 meses. Deve-se acrescentar que a forma de coleta com angulação proposta no presente estudo é inédita, não tendo sido descrita anteriormente.

\section{Discussão}

Para que a coleta seja bem sucedida, a contenção precisa permitir que o corpo da serpente fique totalmente imóvel, sendo necessário que o animal seja contido por indivíduos experientes. O procedimento também pode ser realizado com o animal anestesiado, como descrito para outras técnicas ${ }^{1-8}$. Por se tratar de um animal peçonhento, a manipulação e a coleta exigem treinamento para minimizar o tempo de contato com o animal não sedado e os riscos de acidentes, tanto para os humanos, quanto para as serpentes. O posicionamento é também de suma importância para detectar anatomicamenteo posicionamento do coração pelo frêmito cardíaco $^{8}$, sendo este procedimentomais simples e menos complexo do que pela análise de folidose. Quando esta última é utilizada, o coração localiza-se entre a sexagésima terceira a sexagésima oitava escamas ventrais ${ }^{1}$, o que pode não ser viável, em boa parte das serpentes com menos de 1 metro de comprimento. A punção cardíaca permite a coleta de um grande volume de sangue, contudo a contenção e os cuidados na velocidade da punção devem ser cuidadosamente respeitados ${ }^{5}$. Assim como nas aves, o risco de desenvolvimento de endocardites ou perfuração de estruturas anatomicamente adjacentes devem ser levados em consideração antes da realização doprocedimento ${ }^{2}$. A adaptação da técnica de punção cardíaca proposta neste trabalho se demonstrou apropriada para serpentes do gênero Crotalus durissus, sendo de fácil realização, prática e rápida, permitindo uma coleta de volume de sangue adequado ${ }^{1-5}$. Estes fatores, associados à possibilidade da realização do procedimento sem anestesia, e ao fato de que nenhum dos cinco animais utilizados apresentou efeitos adversos logo após a coleta e mantiveram-se sem quaisquer problemas advindos da punção durante um período de acompanhamento de seis meses, tornam a técnica modificada de punção cardíaca com angulação de $90^{\circ}$ altamente recomendável em situações da clínica de animais silvestres. A confirmação da ausência de lesões permanentes causadas pela punção poderiam ser confirmadas por uma avaliação histológica do órgão cardíaco, no entanto a eutanásia dos animais não estava prevista neste trabalho.

\section{Conclusão}

A técnica descrita neste trabalho apresenta uma alternativa prática e segura para a coleta de sangue através de punção cardíaca em cascavéis, possibilitando a retirada de volumes de sangue em torno de $1 \mathrm{~mL}$ sem efeitos deletérios.

\section{Agradecimentos}

A FAPEMIG, e ao apoio a pesquisa pela Universidade Severino Sombra.

\section{Nota}

Aprovações do comitê de ética USS/CEUA ${ }^{\circ}$ 010/2014, e do Sistema Nacional de Autorização e Informação da Biodiversidade Número: 46529-1.

\section{Referências}

1. Silva JCR. Tratado de animais selvagens-medicina veterinária: Editora Roca; 2007.

2. Neves Júnior J, Machado R, Pires J, Lauriggio A, Passos A, Couto A. Descrição de uma nova técnica para punção venosa em serpentes, através do estudo em jibóias (boa constrictor). Revista da Universidade Rural - Série Ciências da Vida. 2006;26(Suplemento).

3.Borges AR, Rios CHV, Lemos M, Vashist U, de Sousa BM. Blood samples from Liophis typhlus (Linnaeus, 1758)(Serpentes, Dipsadinae) by cardiac puncture without surgical procedures. Revista Brasileira de Zoociências. $2014 ; 15(1,2,3)$

4. Fudge AM. Laboratory medicine: avian and exotic pets: Elsevier Health Sciences; 2000.

5. Gonçalves G. Manual de emergência em aves. São Paulo: Med Vet. 2010.

6. Divers SJ, Mader DR. Reptile medicine and surgery: Elsevier Health Sciences; 2005.

7. Silva MIAD. Alterações hematológicas e bioquímicas em uma população de Phrynops geoffroanus (Schweigger, 1812) em reposta a estressores ambientes. UNESP; 2011.

8. Jepson L. Clínica de animais exóticos referência rápida. Rio de Janeiro: Elsevier, 2010. 